\title{
Propagation Modelling Using Integral Equation Methods to Enable Co-Existence and Address Physical Layer Security Issues in Cognitive Radio
}

\author{
Eamonn O. Nuallain \\ Centre for Telecommunications Value Chain Research, Trinity College Dublin, Dublin, Ireland \\ E-mail:eon@cs.tcd.ie \\ Received October 14, 2010; revised November 6, 2010; accepted November 8, 2010
}

\begin{abstract}
In this paper it is envisaged that cognitive radios (CRs) consult a supporting network infrastructure for permission to transmit. The network server either grants or rejects these requests by estimating, from the CRs geo-location and antenna features, the likely impact its transmission would have on incumbents and other CR devices. This decision would be based on a real-time radio environment map [1] which would be kept up to date with readings from CRs, sensors and dynamic radio propagation prediction. By this means coexistence with incumbents and other CRs can be satisfied. It is maintained here that integral-equation (IE)—based algorithms are suitable candidates for the propagation engine given their 'automatic' nature and that they can be implemented to give results arbitrarily close to the exact numerical solution. IE methods based on the Fast Multipole Method are examined as a likely route to achieve the accuracy and speed necessary for real-time propagation mapping. It is concluded that the results obtained using one of the most recent of these, the Field Extrapolation Method (FEXM) [2], are promising for rural/suburban profiles and could serve to enable coexistence, for example, in IEEE802.22 networks. It is also explained how dynamic propagation prediction can address some fundamental security threats to CR networks.
\end{abstract}

Keywords: Cognitive Radio, Propagation Modeling, Integral Equations

\section{Introduction}

Cognitive Radio (CR) is a radio technology that refers to the ability of a radio device to sense/learn the communication parameters of its environment and adapt its transmissions accordingly. The ability of a radio device to do this with sufficient sophistication and agility will enable such devices to transmit in underutilized licensed bands without affecting the communications of the licensee.

CR presents the radio community with brand new challenges. Chief among these is the ability of these devices to co-exist with the incumbent operators and other CRs without causing harmful interference.

Real-time radio environment mapping offers a means by which this problem can be resolved. 'The Radio Environment Map (REM) itself is an abstraction of realworld radio scenarios; it characterizes the radio environment of CRs in multiple domains, such as geographical features, regulation, policy, radio equipment capability profile and radio frequency emissions' [1] —in short it is a multidimensional map of what is happening in the radio environment in multiple domains. The information needed to create the signal strength portion of this realtime map would be generated by a radio-propagation prediction algorithm and interpolated/corrected by signalstrength readings which are relayed to the server by CR devices and sensors.

The signal-strength portion of the REM server would then essentially act as a set of 'traffic lights' that a CR device would consult each time it wished to transmit. The effects of the requested transmission could be immediately computed based on the GPS position of the $\mathrm{CR}$ and the intended recipient using the propagation predictor and a digital terrain database hosted on the REM. On the basis of whether or not this transmission would adversely affect incumbents or other CRs a decision would be made by the server whether or not to grant permission to transmit and what the appropriate power limitations should be. This is an explicit solution to the Hidden Node Problem. 
To this end a highly accurate and robust propagation predictor capable of operating in a dynamic environment is needed.

\section{Propagation Prediction}

Propagation prediction over terrain has received scant attention in the academic literature in recent years. This contrasts sharply with the attention it received in the eighties and early nineties with the widespread deployment of $2 \mathrm{G}$ cellular systems; the industry generally settling for sub-optimal models such as that of Hata, Walfisch-Igekami and simple Ray-Tracing combined with basic diffraction models such as Knife-Edge Diffraction with which to plan their networks.

The reasons for this were twofold: Firstly, highly accurate models are unnecessary for 2G and 3G networks. Except in circumstances where terrain topology would make it obvious to an experienced engineer where not to place transmitters, large-scale fading would be present regardless of transmitter location and so there is little need for its precise prediction. The impact of these large-scale fades on service quality is borne by the user, which for voice and SMS is largely tolerable if overall coverage is good.

Secondly, more sophisticated models, such as those based on IE methods, were at this time underdeveloped and computationally too expensive.

Furthermore there is no need for dynamic propagation prediction for these networks. Propagation prediction is, in the main, a once-off process concerned with base stations only and is performed at the time of network rollout.

It is for these reasons that IE methods have largely remained the subject of scattering prediction from small and medium-sized objects despite offering inherent advantages over other methods in propagation prediction over terrain.

These advantages are: Given that the IE formulation is the exact solution to the coverage problem there is no need to reduce the problem to a number of canonical ones. All electromagnetic phenomena (reflection, diffraction etc.) are encapsulated in a single formulation. In this sense IE methods can be said to be "automatic" - that is, they can be directly applied to an arbitrary terrain database without human intervention and without pre- processing and so are suitable for addressing dynamic propagation prediction problems.

It is generally envisaged that CR networks will largely be land-based and operate over distances of less than a few kilometres. It is well known that the large-scale fading signal can vary in the order of tens of decibels over much shorter distances at frequencies of a few hundred megahertz and upwards. To set reliable power limitations when granting permission to transmit it is thus necessary to use a propagation model which predicts large-scale fading which is an inherent feature of IE methods.

Because IE methods are based on the exact numerical formulation of the propagation problem, more reliable numerical error margins can be established.

The main drawback of IE methods has been their computational cost. This has been overcome by theoretical developments and by advances in computer technology to the point where coverage results within approx. $8 \mathrm{~dB}$ of measurement results for circa. $10 \mathrm{Km}$ two-dimensional rural profiles have been obtained on a standard desktop at sub-second speeds [2].

The formulation of the problem to be solved as an IE is intuitively more acceptable than a differential equation formulation. This eases the route to understanding and physically inspired development.

For these reasons it is proposed that IE-based algorithms form the basis for the real-time mapping of the radio environment for $\mathrm{CR}$ in order to provide the degree of reliability necessary in decision making regarding transmissions and, as we shall see presently, in addressing some fundamental Physical-Layer security issues.

\section{Security}

Physical Layer problems such as malfunctioning nodes and intentional jamming can be addressed in the following manner. Central to this discussion is the fact that the PU signal is unique to each topology and extremely difficult to replicate physically outside of the vicinity of the primary transmitter. It is in effect a digital signature.

By comparison of collected RSS data from CR nodes and sensors with the predicted PU signal strength identification of malfunctioning nodes and/or security threats is enabled.

To illustrate this concept let us consider the scenario of an intentional jamming attack. From the outset the PU signal strength map as a function of location has been established using a propagation predictor initially derived from its location, a digital terrain database and the maximum radiated power which are obtained from the licensing authority or 'learned' by the network. This map will have been refined with readings from sensors and CR devices. This is our "pre-established" PU signal strength map. During operation it is then observed that a significant number of nodes are reporting the presence of a PU signal. We may then check if the reported PU signal strength values versus location deviate from the pre-established signal strength versus location values of the PU. If so then we have grounds for suspecting that an intentional jamming attack or a primary receiver jam- 
ming attack is taking place. The latter is a passive jamming attack where a malicious entity has unsuspecting devices outside of the coverage region of the PU direct their transmissions to it, which itself is located within the coverage region of the PU. The result is harmful interference to the PU especially in regions of low signal strength. Grounds for suspecting a jamming attack are reinforced if the PU signal is reported to be present in a locality to the contraindication of nodes which are at other locations within the coverage region of the PU. The region of highest "PU" signal strength will indicate the location of the offending device.

Dealing with malfunctioning nodes would be performed in a similar manner, made simpler however, by there being almost always nodes present to contraindicate its readings which may not be the case with an intentional jamming attack.

\section{Integral Equation Methods}

The Field Extrapolation Method (FEXM) is proposed here as the basis for the propagation functionality of the REM server described above- that is the core propagation engine.

In 1991 Prof. J. B. Andersen et al. published the first paper on IE-based coverage prediction for terrain [3]. In it they modelled the terrain profiles they examined as two-dimensional perfect magnetic conductors. The results they obtained were compared with measurements and results given by the Hata model.

It was clear that the results given by the unmodified IE method were superior to those given by the Hata model in terms of standard deviation from measured results and that it predicted the large-scale fading signal very well. However the computational complexity of the problem was prohibitive being of the order $O\left(N^{2}\right)$ where $N$ is sub-wavelength in dimension.

This prompted efforts to reduce the computational complexity of the problem to enable the timely generation of results with a minimal compromise in accuracy.

According to Peterson [4] the Fast Multiple Method [5] or variations thereof, "appear to offer the most efficient possibilities yet proposed for the accurate numerical analysis of electrically large geometries where $N$ may be far greater than $10^{4,}$.

The evolution of the FEXM from the Fast Multipole Method is traced here via other published methods which will provide a means for critical analysis and hopefully provide the reader with clues on how it may be further enhanced - perhaps in conjunction with other methods.

\subsection{The Electric Field Integral Equation}

The problem is treated as two-dimensional $T M_{Z}$, the surface is taken to be a perfect electrical conductor (PEC) and forward scattering is assumed - that is all radiation is taken to propagate away from the transmitter. These two assumptions are justifiable for the case of grazing incidence transmitter radiation which is predominantly the case for the terrain profile examined here. All are simplifying and not limiting assumptions.

The surface is impinged by a monochromatic $T M_{Z}$ polarized cylindrical wave of wave number $\beta$ emanating from an infinite, unit current carrying wire of negligible cross-section, placed a distance above and transverse to the terrain profile. A time variation of $e^{j \omega t}$ is assumed and suppressed.

An electric current $J$ is induced on the surface, which satisfies the Electric Field Integral Equation (EFIE):

$$
E^{I}(\rho)=\frac{\beta \eta}{4} \int_{S} J\left(\rho^{\prime}\right) H_{0}^{(2)}\left(\beta\left|\rho-\rho^{\prime}\right|\right) d s^{\prime}
$$

$r$ and $r^{\prime}$ are vectors whose end-points are respectively the scattering and receiving points $s \in S . E(r)$ is the source electric field incident on the surface at the point given by $r$.

$\eta$ is the wave impedance of the medium through which the radiation propagates and $H_{0}^{(2)}$ is a zero order Hankel function of the second kind which is the Green's function for the problem. The surface is discretized into $N$ equal sized sampling intervals of length $\Delta s$ with centre-points indicated by the vectors $r_{i}$ and $r_{j}$ depending on whether they are scattering or receiving intervals respectively. Using the Method of Moments with unit pulse basis functions and Dirac-delta weighting functions we get the following matrix relation:

$$
E=Z J
$$

where:

$$
\begin{aligned}
& E_{i}=E^{I}\left(\rho_{i}\right) \\
& Z_{j i}=\Delta s \frac{\beta \eta}{4} H_{0}^{(2)}\left(\left|\rho-\rho^{\prime}\right|\right) \\
& Z_{j j}=\Delta s \frac{\beta \eta}{4}\left(1-j \frac{2}{\pi} \ln \left(\frac{1.781 \beta \Delta s}{4 e}\right)\right) \\
& J_{j}=J\left(\rho_{j}\right)
\end{aligned}
$$

$E$ and $J$ are column vectors of length $N$. $Z$, known as the impedance matrix, is $N \times N$ and symmetric. The elements in the strictly lower triangle of $Z$ correspond to forward-scattering and those in the strictly upper triangle to back-scattering. The diagonal elements correspond to the self-interaction of the sampling intervals. On the assumption of forward scattering, which is equivalent to setting the strictly upper triangular elements of $E$ to zero, $J$ is determined by forward substitution: 


$$
E_{i}=\sum_{j=1}^{j \leq i} J_{j} Z_{j i} \quad \text { for } \quad i=1, \cdots, N
$$

The order of complexity of determining $J$ is $O\left(N^{2}\right)$. The total field at points above the surface is then the sum of the field from the source and the field scattered by the surface.

The surface is divided into groups each containing $M$ sampling intervals. There are then $N / M$ such groups.

A point of note, the importance of which will become apparent later, is that this equation converges and gives almost identical results if the Hankel function is replaced with its far-field approximation. Why this is so is not clear but it is a very useful observation given that the far-field form is much easier to manipulate algebraically.

\subsection{The Fast Multipole Method}

The $N$ integration intervals on the surface are grouped such that each group comprises $M$ integration intervals. The number of groups is then $N / M$. Letting $G_{l}$ and $G_{l^{\prime}}$ denote groups with centre points $l$ and $l$ respectively. The impedance matrix may then be written:

$$
[Z]=[B]^{T}[A][B]
$$

where:

$$
a_{n m}\left(l, l^{\prime}\right)=H_{n-m}^{(2)}\left(\beta\left|\rho_{l}-\rho_{l^{\prime}}\right|\right) e^{j(n-m) \varphi_{l l^{\prime}}}
$$

and

$$
b_{j}\left(j, l^{\prime}\right)=\mathfrak{J}_{j}\left(\beta\left|\rho_{l}-\rho_{l^{\prime}}\right|\right) e^{j n \varphi_{j l^{\prime}}}
$$

$\varphi_{l l^{\prime}}$ is the angle $\rho_{l}-\rho_{l^{\prime}}$ makes with the horizontal and similarly for $\varphi_{j l^{\prime}}$.

Then:

$$
E^{I}\left(\rho_{j}\right)=\frac{\beta \eta}{4} b_{l^{\prime} j}^{T} \sum_{l=1, l=l^{\prime}}^{N / M} a_{l l^{\prime}} \sum_{i \in G_{l}} b_{i l} J\left(\rho_{i}\right) \Delta s_{i}
$$

where $j \in G_{l^{\prime}}$ and $l^{\prime}=1, \cdots, \frac{N}{M}$

The complexity of this equation can be substantially reduced if $[A]$ can be diagonalised.

To this end we substitute the definitions for $a$ and $b$ given in (5) so that this equation becomes:

$$
\begin{aligned}
H_{0}^{(2)}\left(\beta\left|\rho_{l}-\rho_{l^{\prime}}\right|\right) & =\sum_{m=-\infty}^{\infty} \mathfrak{I}_{m}\left(\beta \rho_{j l^{\prime}}\right) e^{j m\left(\varphi_{j l^{\prime}}-\pi\right)} \\
& \times \sum_{n=-\infty}^{\infty} H_{m-n}^{(2)}\left(\beta \rho_{l^{\prime}}\right) e^{-j(m-n) \varphi_{I^{\prime}}} \mathfrak{I}_{m}\left(\beta \rho_{i l}\right) e^{-j n \varphi_{i l}}
\end{aligned}
$$

The inner summation is a convolution of two DFTs and hence can be expressed as a product of two functions if their DFTs are known. The DFT of $H_{n}^{(2)}(x) e^{j n \varphi}$ does not exist since
$H_{n}^{(2)}(x) \rightarrow \infty$ as $n \rightarrow \infty$. However we can truncate the inner summation since it converges.

Via the identity:

$$
\mathfrak{I}_{m}\left(\beta \rho_{l^{\prime} j}\right) e^{i m \varphi_{l^{\prime} j}}=\frac{1}{2 \pi} \int_{0}^{2 \pi} e^{j \beta \rho_{l^{\prime} j} \cos \left(\alpha-\varphi_{l^{\prime}}\right)+j m\left(\alpha-\frac{\pi}{2}\right)} d \alpha
$$

we can write:

$$
H_{0}^{(2)}\left(\beta \rho_{i j}\right)=\frac{1}{2 \pi} \int_{0}^{2 \pi} b_{l^{\prime} j}(\alpha) \alpha_{l l^{\prime}} b_{i l}(\alpha) d \alpha
$$

where:

$$
\begin{aligned}
& a_{l l^{\prime}}(\alpha)=\sum_{p=-P}^{P} H_{0}^{(2)}\left(\beta \rho_{l l^{\prime}}\right) e^{-j p\left(\varphi_{\left.l^{\prime} l^{\prime}+\alpha-\frac{\pi}{2}\right)}\right.} \\
& b_{i j}(\alpha)=e^{j \beta \rho_{i j} \cos \left(\alpha-\varphi_{i j}\right)}
\end{aligned}
$$

Now using Equation (8) in Equation (6) we have:

$$
E^{I}\left(\rho_{j}\right)=\frac{\beta \eta}{8 \pi} \int_{0}^{2 \pi} b_{l^{\prime} j}(\alpha) \sum_{i=1, i=l^{\prime}}^{N / M} a_{l l^{\prime}}(\alpha) \sum_{i \in G_{l}} b_{i l}(\alpha) J\left(\rho_{i}\right) \Delta s_{i}
$$

Replacing the outer integral with a $Q$-point summation gives:

$$
E^{I}\left(\rho_{j}\right)=\frac{\omega \mu_{0}}{4 Q} \sum_{q=1}^{Q} b_{l^{\prime} j}(\alpha) \sum_{i=1, i=l^{\prime}}^{N / M} a_{l l^{\prime}}(\alpha) \sum_{i \in G_{l}} b_{i l}(\alpha) J\left(\rho_{i}\right) \Delta s_{i}
$$

This is the Fast Multipole Method (FMM) formulation for the above described problem.

\subsection{The Fast Far-Field Approximation}

The Fast Far-Field Approximation (FAFFA) [6] can be obtained from the FMM in the following way [7]:

$a_{l l^{\prime}}(\alpha)$ diverges as $P \rightarrow \infty$ This does not occur if we use the far-field approximation of the Hankel function which , as noted previously, does not affect the convergence of the integral:

$$
H_{0}^{(2)}(x) \approx \sqrt{\frac{2 j}{\pi x}} e^{-j\left(x-\frac{\pi}{2}\right)}
$$

This allows one to write:

$$
\begin{array}{r}
a_{l l^{\prime}}(\alpha) \approx \sqrt{\frac{2 j}{\pi \beta \rho_{l l^{\prime}}}} e^{j \beta \rho_{l l^{\prime}}} \sum_{p=-P}^{P} e^{-j p\left(\varphi_{l l^{\prime}}-\alpha\right)} \\
=\sqrt{\frac{2 j}{\pi \beta \rho_{l l^{\prime}}}} \frac{\sin \left(\left(P+\frac{1}{2}\right)\left(\varphi_{l l^{\prime}}-\alpha\right)\right)}{\sin \left(\frac{1}{2}\left(\varphi_{l l^{\prime}}-\alpha\right)\right)} \\
\rightarrow \frac{\pi\left(\alpha-\varphi_{l l^{\prime}}\right)}{\sin \left(\frac{1}{2}\left(\alpha-\varphi_{l l^{\prime}}\right)\right)} \delta\left(\alpha-\varphi_{l l^{\prime}}\right) ; P \rightarrow \infty
\end{array}
$$


where $\delta(\alpha)$ is the Dirac-delta function.

Hence:

$$
\begin{aligned}
H_{0}^{(2)}\left(\rho_{i j}\right) & \approx \sqrt{\frac{2 j}{\pi \beta \rho_{l l^{\prime}}}} e^{j \beta \rho_{l l^{\prime}}} \\
& \times \int_{0}^{2 \pi} b_{l l^{\prime} j} \frac{\pi\left(\alpha-\varphi_{l l^{\prime}}\right)}{\sin \left(\frac{1}{2}\left(\alpha-\varphi_{l l^{\prime}}\right)\right)} \delta\left(\alpha-\varphi_{l l^{\prime}}\right) b_{i l}(\alpha) d \alpha
\end{aligned}
$$

Via L’Hopital's Rule we get:

$$
H_{0}^{(2)}\left(\rho_{i j}\right) \approx \sqrt{\frac{2 j}{\pi \beta \rho_{l l^{\prime}}}} e^{j \beta \rho_{l l^{\prime}}} b_{l^{\prime} j}\left(\varphi_{l l^{\prime}}\right) b_{i l}\left(\varphi_{l l^{\prime}}\right)
$$

The discrete form of the EFIE is separated into its near and far-field regions giving:

$$
\begin{aligned}
E^{I}\left(\rho_{j}\right)= & \sum_{G_{l^{\prime}} \in F F} e^{j \beta \Delta_{i l} \cos \left(\varphi_{I l^{\prime}}-\varphi_{i l}\right)} \\
& \times \sqrt{\frac{2 j}{\pi \beta \rho_{I l^{\prime}}}} e^{j \beta \rho_{I l^{\prime}}} \sum_{j \in G_{l^{\prime}}} e^{j \beta \Delta_{i l} \cos \left(\varphi_{I I^{\prime}}-\varphi_{l j}\right)} J\left(\rho_{j}\right) \Delta s^{\prime} \\
& +\sum_{j \in G_{I^{\prime}} \in N F} H_{0}^{(2)}\left(\rho_{j i}\right) J\left(\rho_{j}\right) \Delta s^{\prime}
\end{aligned}
$$

where $N F$ and FF denote the near and far-field components of the scattered field respectively. In like manner with the FMM, the summations may be viewed as aggregation (at the scattering group $G_{l}$ ), translation (between groups) and disaggregation (at the 'receiving' group $G_{l^{\prime}}$ ) stages in the order in which they are given above. This is the Fast Far-Field Approximation. Computational speed up is achieved since the result of the disaggregation stage can be stored and reused.

\subsection{The Tabulated Interaction Method}

With the Tabulated Interaction Method [8] the integration intervals in a group are taken to be collinear and the aggregation and disaggregation stages referred to in Lu and Chew's paper [6] are performed for a range of angles of incidence and reflection, $\varphi_{l l^{\prime}}-\varphi_{l^{\prime} j}$ and $\varphi_{I I^{\prime}}-\varphi_{i l}$ respectively, creating a lookup table which is then used in performing the overall summation.

Using the approximation:

$$
J\left(\rho_{i}\right) \approx E^{S}\left(\rho_{l}\right) J_{\text {Plane }}\left(\rho_{i}, \varphi_{l l^{\prime}}-\varphi_{i l}\right)
$$

where $J_{\text {Plane }}\left(\rho_{i,}, \varphi_{I I^{\prime}}-\varphi_{i l}\right)$ is the surface current induced at the point denoted by $\rho_{i}$ as a result of a unit amplitude plane wave incident on the surface at an angle of $\varphi_{l l^{\prime}}-\varphi_{i l}$.

Then:

$$
\begin{aligned}
E^{I}\left(\rho_{l^{\prime}}\right)= & \sum_{G_{l} \in F F}^{G_{l}<G_{l^{\prime}}} H_{0}^{(2)}\left(\rho_{l l^{\prime}}\right) e^{j \beta \Delta_{j l} \cos \left(\varphi_{l l^{\prime}}-\varphi_{i l}\right)} E^{S}\left(\rho_{l}\right) \\
& \times \sum_{j \in G_{l}} J_{\text {Plane }}\left(\rho_{i}, \varphi_{l l^{\prime}}-\varphi_{i l}\right) e^{j \beta \Delta_{j l} \cos \left(\varphi_{l l^{\prime}}-\varphi_{l^{\prime}}\right)} \Delta s^{\prime}( \\
& +\sum_{j \in N F} J_{j} H_{0}^{(2)}\left(\rho_{i j}\right) \Delta s^{\prime}
\end{aligned}
$$

The inner summation (or disaggregation stage) having been pre-computed can be accessed via a look-up table thereby speeding up the solving process. Equation (14) can be written more accurately as:

$$
H_{0}^{(2)}\left(\rho_{i j}\right) \approx H_{0}^{(2)}\left(\rho_{I I^{\prime}}\right) b_{l^{\prime} j}\left(\varphi_{I l^{\prime}}\right) b_{i l}\left(\varphi_{I I^{\prime}}\right)
$$

It is also noted that because of the nature of the problem-land-based transmission-the angles $\varphi_{l l^{\prime}}-\varphi_{l^{\prime} j}$ and $\varphi_{l l^{\prime}}-\varphi_{i l}$ will generally be small-especially in the region near the observation point.

Then from Equations (17) and (18), making no distinction between the groups in the near and far-field, we get the following approximation:

$$
\begin{aligned}
E^{I}\left|\rho_{l^{\prime}}\right| \approx & \sum_{G_{l}=0}^{G_{l}<G_{I^{\prime}}} H_{0}^{(2)}\left(\rho_{l^{\prime}}\right) \sum_{j \in G_{I^{\prime}}} e^{j 2 \beta \Delta_{i l}} J\left(\rho_{i}\right) \Delta s^{\prime} \\
& +\sum_{j \in G_{I^{\prime}}}^{G_{l}=G_{I^{\prime}}} J_{j} H_{0}^{(2)}\left(\rho_{i j}\right) \Delta s^{\prime}
\end{aligned}
$$

\subsection{The Field Extrapolation Method}

The TIM has shown that interactions between groups may be considered as being due to plane- waves. Where the groups are approximated as being collinear then it follows that the surface current may be approximated as being constant amplitude sinusoids. This a-priori assumption of the form of the induced surface current over a group can be used to circumvent the aggregation and disaggregation stages of the FAFFA and the creation of a look-up table in the TIM. The resulting algorithm is very simple, very fast, has negligible memory requirements and yields results of similar accuracy to the aforementioned.

It has been shown how the algorithm has its conceptual roots in all three of the above.

These are:

1) The fundamental concept of effectively grouping integration intervals into equal sized regions has its origin in the FMM.

2) The concept of summing phase-shifted values of the surface current at the group centre during "aggregation" and performing the reverse procedure at the "disaggregation" stage has its roots in the FMM and the FAFFA.

3) The concept that group-group interactions can be treated as being due to plane wave interactions has its 
roots in the TIM.

All three of these concepts form the basis for the FEXM. What follows is a heuristic derivation of the FEXM starting with the FAFFA where the contributions of the above concepts become clear.

From Equation (19), but treating near-field interactions as though they are due to far-fields except at the receiving group $G_{l}$ we get:

$$
\begin{aligned}
E^{I}\left(\rho_{i}\right) & =\sum_{G_{l}}^{G_{l}<G_{I^{\prime}}} e^{j \beta \Delta_{\Delta_{i}} \cos \left(\varphi_{I^{\prime}}-\varphi_{i l}\right)} \\
& \times \sqrt{\frac{2 i}{\pi \beta \rho_{l l^{\prime}}}} \sum_{j \in G_{I^{\prime}}} e^{j \beta \Delta_{i l} \cos \left(\varphi_{I^{\prime}}-\varphi_{l^{\prime}}\right)} J\left(\rho_{j}\right) \Delta s^{\prime}
\end{aligned}
$$

This equation in itself does not converge to give the correct solution. This is because the near-field interactions have not been treated as such and so the above approximation is too crude. The near-field interactions are those which have the greater bearing on convergence. This corresponds to the disaggregation stage in the above equation. Because the angle $\varphi_{I I^{\prime}}-\varphi_{i l}$ and $\varphi_{I l^{\prime}}-\varphi_{l^{\prime} j}$ will generally be small and the aggregation phase can be combined with the translation phase with comparatively little loss in accuracy, we get:

$$
\begin{aligned}
E^{I}\left(\rho_{i}\right) & =\sum_{G_{l}}^{G_{l}<G_{l^{\prime}}} \sqrt{\frac{2 i}{\pi \beta \rho_{I l^{\prime}}}} e^{j \beta\left(\rho_{l^{\prime}}+\Delta_{i l}\right)} \sum_{j \in G_{l^{\prime}}} e^{j \beta \Delta_{i l}} J\left(\rho_{j}\right) \Delta s^{\prime} \\
& \approx \sum_{G_{l}}^{G_{l} \leq G_{I^{\prime}}} H_{0}^{(2)}\left(\rho_{l I^{\prime}}\right) \sum_{j \in G_{l^{\prime}}} e^{j \beta \Delta_{i l}} J\left(\rho_{j}\right) \Delta s^{\prime}
\end{aligned}
$$

The process of disaggregation is performed by the phase shifting operator $e^{j \beta \Delta_{i l}}$. It is now proposed that this sensitive process is performed in both amplitude and phase. This is achieved using the Green's function for the problem, namely the Hankel function.

The resulting equation is then:

$$
E^{I}\left(\rho_{j}\right)=\sum_{G_{l}}^{G_{l}<G_{I^{\prime}}} H_{0}^{(2)}\left(\rho_{l l^{\prime}}\right) \sum_{j \in G_{l^{\prime}}} H_{0}^{(2)}\left(\rho_{i j}\right) J\left(\rho_{j}\right) \Delta s^{\prime}
$$

As was shown in the TIM, the surface current can be modelled as having been induced by a plane wave. If furthermore we assume that the amplitude of this current is approximately constant over a group then we can write:

$$
\begin{aligned}
E^{I}\left(\rho_{l}\right)= & \sum_{G_{l}}^{G_{l} \leq G_{l^{\prime}}} H\left(\rho_{l l^{\prime}}\right) J\left(\rho_{l}\right) \\
& \times \sum_{j \in G_{l^{\prime}}} H_{0}^{(2)}\left(\rho_{i j}\right) e^{-j \beta \Delta_{j i} \cos \left(\theta_{l^{\prime}}\right)} \Delta s^{\prime}
\end{aligned}
$$

If, as in the case of the TIM, groups can be considered to be flat the inner summation will be a constant where the phase shift $\theta_{l^{\prime}}$ is incorporated into the current phasor. This latter approximation is reasonable since we are not interested in small-scale fading. Hence:

$$
E^{I}\left(\rho_{l}\right)=\mathrm{K} \sum_{G_{l}}^{G_{l} \leq G_{I^{\prime}}} H_{0}^{(2)}\left(\rho_{l l^{\prime}}\right) J\left(\rho_{l}\right) \Delta s^{\prime}
$$

where

$$
\mathrm{K}=\sum_{i \in G_{l}} H_{0}^{(2)}\left(\rho_{i l}\right) e^{-j \beta \Delta_{i l}} \Delta s^{\prime}
$$

This is a somewhat different result for $\mathrm{K}$ to that obtained in [2] but the absolute value is similar. The phase is arbitrary and can be absorbed into the current phasor without any appreciable difference in the result for field coverage at points $t$ above the surface which, by similar analysis is:

$$
E^{T}\left(\rho_{t}\right)=E^{I}\left(\rho_{t}\right)+\mathrm{K} \sum_{G_{l}}^{G_{l} \leq G_{l^{\prime}}} H_{0}^{(2)}\left(\rho_{l t}\right) J\left(\rho_{l}\right) \Delta s^{\prime}
$$

From the above analysis it can be seen that the FEXM achieves its accuracy by using the Green's function as the operator for disaggregation and its speed by virtue of a-priori assumption of the form of the surface current over a group. In this way the need to perform computationally expensive group-specific disaggregation or the creation of look-up tables is obviated. The algorithm is simple, lends itself easily to application on a digital terrain database and like the EFIE this algorithm is parallelizable.

\section{Results}

The following results were introduced in [9] and, it is hoped, will serve to illustrate the preceding discussion. The FEXM is applied at $435 \mathrm{MHz}$ to a $7.85 \mathrm{Km}$ semirural terrain profile in Hadsund, Northern Denmark shown in Figure 1. In Figure 2, a comparative plot of results given by the FEXM, EFIE and measurements is given. The transmitter is at an elevation of $16.4 \mathrm{~m}$. All measurements and computational results are taken at 2.4 $\mathrm{m}$ above the surface. All computations are performed on a $2.2 \mathrm{GHz}$ Pentium 4 processor. $10 \mathrm{~m}$ group sizes are used to provide as accurate as possible a comparison with measurements (which were taken every $10 \mathrm{~m}$ ). The execution time is $1.35 \mathrm{sec}$ yielding a circa. $7.5 \mathrm{~dB}$ standard deviation with respect to measurements. Much larger group sizes can be used for these types of profiles without greatly compromising accuracy. The execution time using $50 \mathrm{~m}$ group sizes is $0.07 \mathrm{sec}$.

In Figure 3, the transmitter is placed $1 \mathrm{~m}$ above the surface, mimicking a CR, at $x=5 \mathrm{Km}$. The effect of the transmission is plotted against location. The execution times are as above. 


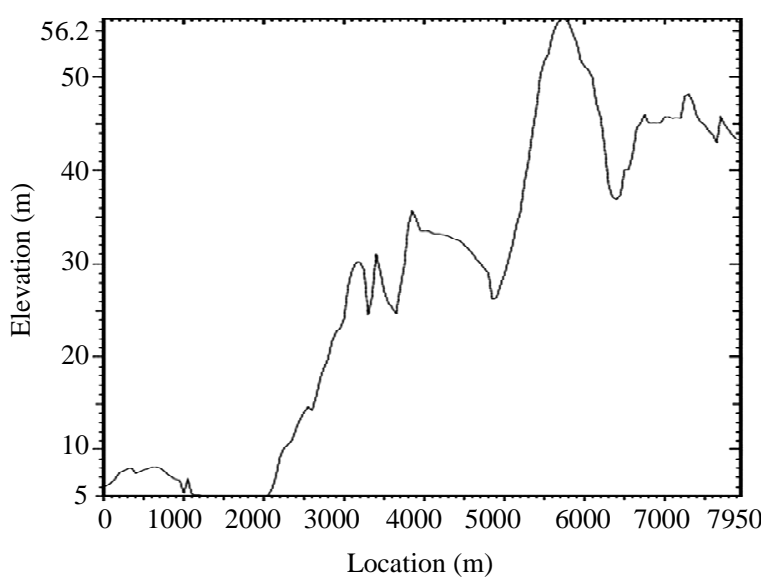

Figure 1. Plot of terrain elevation versus location.

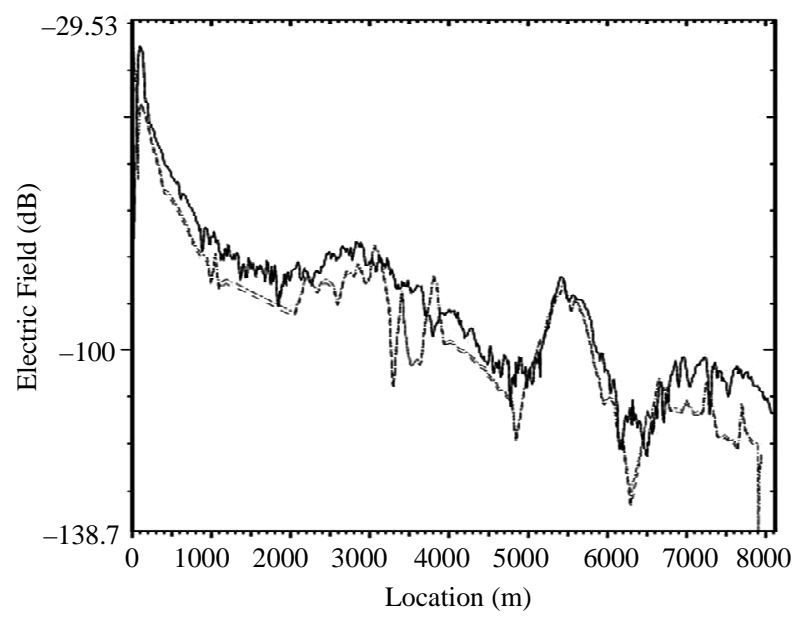

Figure 2. Comparative plot of Electric Field Strength at $\mathbf{2 . 4}$ $m$ above the surface versus location as given by the EFIE (dot-dash), Measurements (solid) and the FEXM (dashed). The transmitter is located at an elevation of $16.4 \mathrm{~m}$ at $\mathrm{x}=\mathbf{0}$. The transmission frequency is $435 \mathrm{MHz}$.

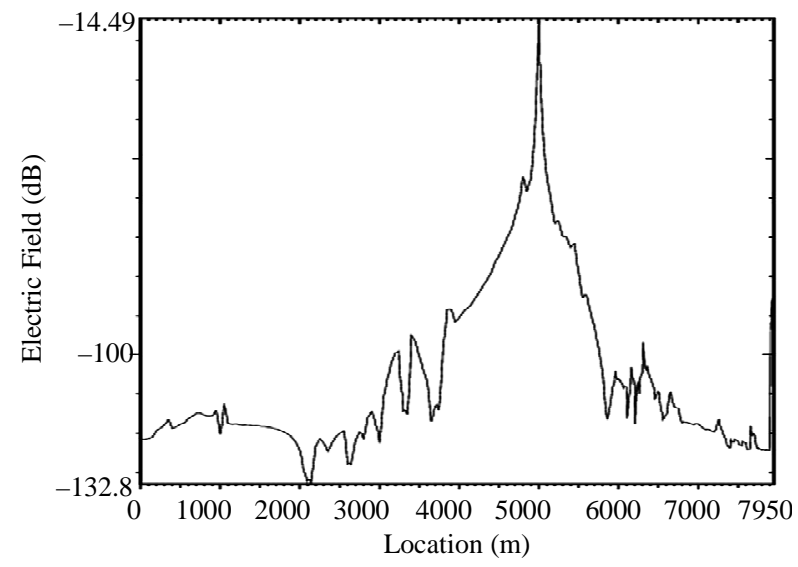

Figure 3. Plot of Electric Field Strength at $2.4 \mathrm{~m}$ above the surface versus location as given by the FEXM for a transmitter placed $1 \mathrm{~m}$ above the surface at $x=5000 \mathrm{~m}$. The transmission frequency is $435 \mathrm{MHz}$.

\section{Conclusions}

In this paper the idea of using a real-time propagation predictor on a supporting server to decide on whether to grant/reject transmission requests of a CR and set appropriate power limitations has been explored. It is necessary that large-scale fading be accurately predicted in order to achieve this functionality. It is argued that IE methods are appropriate for this task because of their 'automatic' nature.

It is likely that network support will be necessary for CR since it is beyond the means of CRs themselves to address the 'Hidden Node Problem'. It is clear that such support will offer other advantages too such as providing a means with which to combat security threats and enabling a reduction in the complexity and consequently the price of CRs themselves.

The paper has focused on developments in fast IE methods based on the FMM as a likely route to an effective IE-based propagation predictor. The most efficient result to-date, the FEXM, has been applied to a number of terrain profiles at different frequencies yielding promising results [2].

It is observed in trials, using the method described in [10], that backscattering is negligible for the profiles examined. This makes it probable that side-scattering does not have a major bearing on coverage results for rural profiles, which if true, means that a full 3D implementation may be unnecessary. The 2-D coverage results are very encouraging. Close agreement with measured/ numerically exact results is observed. It is clear from the desktop execution times that the possibility of obtaining a real-time predictor for rural profiles on a powerful server is real.

At this point in time the problem of obtaining accurate and timely coverage results for urban areas using IE methods has not been examined in the literature. This will undoubtedly be a challenging task. Indeed, the accelerated methods described above may not give the necessary speed-up and accuracy needed, in which case multilevel algorithms of the type described in [11] or parallelization are means worthy of consideration.

\section{Acknowledgements}

The author would like to thank Prof. J. B. Andersen of Aalborg University in Denmark for his kind permission to use the measurement results given in this paper.

\section{References}

[1] Y. Zhao, B. Le and J. Reed, "Network Support: The Radio Environment," In: B. Fette, Ed., Cognitive Radio Technology, Elsevier, Amsterdam, 2006. 
[2] E. O. Nuallain, “An Efficient IE-Based Propagation Model," IEEE Transactions on Antennas and Propagation, Vol. 53, No. 5, May 2005, pp. 1836-1841. doi:10.1109/TAP.2005.846812

[3] J. T. Hviid, J. B. Andersen, J. Toftgard and J. Bojer, “Terrain-Based Propagation Model for Rural Areas-An Integral Equation Approach,” IEEE Transactions in Antennas and Propagation, Vol. 43, No. 1, January 1995, pp. 41-46. doi:10.1109/8.366349

[4] A. F. Peterson, S. L. Ray and R. Mittra, "Computational Methods for Electromagnetics,” IEEE Press, New York, 1998, pp. 37-45.

[5] R. Coifman, V. Rokhlin and S. Wandzura, "The Fast Multipole Method for the Wave Equation: A Pedestrian Description,” IEEE Antennas and Propagation Magazine, Vol. 35, No. 3, 1993, pp. 7-12. doi:10.1109/74.250128

[6] C. C. Lu and W. C. Chew, "Fast Far Field Approximation for Calculating the RCS of Large Objects," Microwave and Optical Technology Letters, Vol. 8, No. 5, 1995, pp. 238-241. doi:10.1002/mop.4650080506

[7] C. Brennan, "Numerical Methods for the Efficient Computation of Electromagnetic Scattering from a Class of
Large Scale Perfect Electrical Conductors,” Thesis, Trinity College, Dublin, 1998.

[8] C. Brennan and P. Cullen, "Tabulated Interaction Method for UHF Terrain Propagation Problems,” IEEE Transactions on Antennas and Propagation, Vol. 46, No. 5, 1998, pp. 738-739. doi:10.1109/8.668921

[9] E. O. Nuallain, “A Proposed Propagation-Based Methodology with Which to Address the Hidden Node Problem and Security/Reliability Issues in Cognitive Radio," International Conference on Wireless Communications, Networking and Mobile Computing, Dalian, 12-14 October 2008, pp. 1-5.

[10] D. Holliday, L. DeRaad and G. St-Cyr, "Forward-Backward: A New Method for Computing Low-Grazing Angle Scattering," IEEE Transactions on Antennas and Propagation, Vol. 44. No. 5, 1996, pp. 722-729. doi:10.1109/8.496263

[11] J. M. Song and W. C. Chew, "Multilevel Fast Multipole Algorithm for Solving Combined Field Integral Equations of Electromagnetic Scattering,” Microwave and Optical Technology Letters, Vol. 10, No 1, 1995, pp. 14-19. doi:10.1002/mop.4650100107 